Данилин А.Н., Курдюмов Н.Н., Кузнецова Е.Л., Рабинский Л.Н. О моделировании деформирования проволочных конструкций спиральной структуры // Вестник Пермского национального исследовательского политехнического университета. Механика. - 2015. - № 4. - C. 72-93. DOI: 10.15593/perm.mech/2015.4.05

Danilin A.N., Kurdumov N.N., Kuznetsova E.L., Rabinsky L.N. Modelling of deformation of wire spiral structures PNRPU Mechanics Bulletin. 2015. No. 4. Pp. 72-93. DOI: 10.15593/perm.mech/2015.4.05

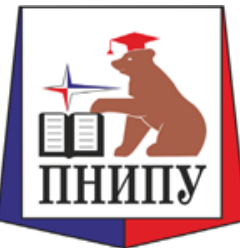

ВЕСТНИК ПНИПУ. МЕХАНИКА

№ 4, 2015

PNRPU MECHANICS BULLETIN

http://vestnik.pstu.ru/mechanics/about/inf/

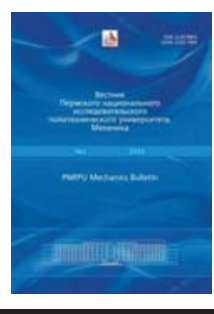

DOI: $10.15593 /$ perm.mech/2015.4.05

УДК 539.313

О МОДЕЛИРОВАНИИ ДЕФОРМИРОВАНИЯ ПРОВОЛОЧНЫХ КОНСТРУКЦИЙ СПИРАЛЬНОЙ СТРУКТУРЫ

\author{
А.Н. Данилин ${ }^{1,2}$, Н.Н. Курдюмов ${ }^{1,2}$, Е.Л. Кузнецова ${ }^{2}$, Л.Н. Рабинский ${ }^{2}$ \\ ${ }^{1}$ Институт прикладной механики РАН, Москва, Россия \\ ${ }^{2}$ Московский авиационный институт (национальный исследовательский университет), \\ Москва, Россия
}

O CTATBE

Получена: 31 июля 2015 г.

Принята: 13 октября 2015 г.

Опубликована: 25 декабря 2015 г.

Ключевые слова:

провод, спиральный зажим, проволочный слой,

энергетическое осреднение,

безмоментная цилиндрическая

оболочка, жесткости,

несущая способность

\section{АННОТАЦИЯ}

Анализ работоспособности воздушных линий электропередачи (ЛЭП) включает в себя расчеты статических состояний и колебаний проводов (и тросов) совместно с арматурой, гасителями колебаний и другими закрепленными на них устройствами. Решение многих из этих задач возможно только при корректном учете внутренней структуры проводов, конструкция которых формируется проволочными слоями (повивами), навитыми друг на друга под разными углами относительно продольной оси. Например, такой учет необходим при проектировании систем энергобезопасности и надежности информационно-телекоммуникационного обеспечения аэродромов, авиационных и ракетных систем, воздушных ЛЭП общего назначения, подверженных интенсивному воздействию ветра, особенно в условиях обледенения.

Из-за сложной структуры проволочных конструкций возникают известные проблемы в оценках их деформаций, жесткостей, несущей способности и пр. Например, изгибная жесткость провода может заметно меняться по мере его деформации, поскольку проволочные слои провода могут проскальзывать относительно друг друга, а отдельные проволоки - перемещаться внутри повивов. Следовательно, величины изгибной и крутильной жесткостей могут изменяться как вдоль оси провода, так и во времени.

В работе предлагается новая модель деформирования проволочных конструкций, подобных проводам ЛЭП. К таким конструкциям относятся не только провода и тросы ЛЭП, но и спиральные зажимы, предназначенные для натяжения, подвески, соединения, защиты и ремонта проводов.

Каждый проволочный повив представляется с позиции энергетического осреднения как эквивалентная по упругим свойствам анизотропная цилиндрическая оболочка, а сам провод или спиральный зажим рассматриваются как система вложенных друг в друга цилиндрических оболочек, между которыми допускается проскальзывание с учетом сил давления и трения. На основе этого подхода получены формулы для определения матриц податливости и жесткости спиральных конструкций. Сформулирована и решена задача о взаимодействии натяжного зажима с внешним повивом провода и исследован механизм передачи усилия с зажима на провод.

(с) ПНИПУ

( С Данилин Александр Николаевич - доктор фризико-математических наук, главный научный сотрудник, e-mail: andanilin@yandex.ru

Курдюмов Николай Николаевич - доцент, e-mail: nick.n.kurdyumov@gmail.com

Кузнецова Елена Львовна - кандидат физико-математических наук, доцент, e-mail: vida_ku@mail.ru

Рабинский Лев Наумович - доктор физико-математических наук, профресcop, e-mail: rabinskiy@mail.ru

Alexander N. Danilin - Doctor of Physical and Mathematical Sciences, Senior Researcher, e-mail: andanilin@yandex.ru Nikolay N. Kurdumov - Associate Professor, e-mail: nick.n.kurdyumov@gmail.com

Elena L. Kuznetsova - Ph.D. in Physical and Mathematical Sciences, Associate Professor, e-mail: vida_ku@mail.ru Lev N. Rabinsky - Doctor of Physical and Mathematical Sciences, Professor, e-mail: rabinskiy@mail.ru 


\title{
MODELLING OF DEFORMATION OF WIRE SPIRAL STRUCTURES
}

\author{
A.N. Danilin ${ }^{1,2}$, N.N. Kurdumov ${ }^{1,2}$, E.L. Kuznetsova ${ }^{2}$, L.N. Rabinsky ${ }^{2}$ \\ ${ }^{1}$ Institute of Applied Mechanics, Russian Academy of Sciences, Moscow, Russian Federation \\ ${ }^{2}$ Moscow Aviation Institute (National Research University), Moscow, Russian Federation
}

\section{ARTICLE INFO}

Received: 31 July 2015

Accepted: 13 October 2015

Published: 25 December 2015

Keywords:

conductor, spiral clamp, wire layer, energy averaging, membrane cylindrical shell,

stiffnesses, bearing capacity

\begin{abstract}
Analysis of power transmission lines (PTL) involves the calculations of static states and vibrations of conductors (and cables) together with spiral accessories, vibration dampers and other devices attached on them. Many of these problems can be properly solved only by taking into consideration the internal structure of the conductors, the design of which is formed by wire layers wound on each other at different angles relative to the longitudinal axis. For example, such reference is required in the design of the systems of power safety and reliability of information-telecommunication supply of aerodromes, aircraft and rocket systems, overhead transmission lines, subject to intense wind, especially in icing conditions.

Due to the complex design of the wire structures the known issues arise in the estimates of their deformations, stiffnesses, bearing capacity, etc. For example, the bending stiffness of the conductor can sufficiently vary as its deformation, since the wire layers may slip relative to each other, and a separate wire is movable within the wire layer. Consequently, the values of stiffnesses can be varied both along the conductor axis and in time.

The paper proposes a new deformation model of wires structures which are similar toPTL conductors. These structures include not only conductors and cables, but spiral clamps intended for tension, suspension, joints, protection and repair of conductors.

On the basis of energy averaging, each wire layer is considered as an elasticallyequivalent anisotropic cylindrical shell, thus a conductor or a spiral clamp are modeled as a system of cylindrical shells nested into each other and interacting by the forces of pressure and friction. Following this approach the formulae for the flexibility and stiffness matrices of spiral structures have been obtained. The problem of interaction of a tension clamp with the external wire layer of a conductor has been formulated and solved. The mechanism of the force transfer from the clamp on the conductor has been investigated.
\end{abstract}

\section{Введение}

В настоящее время задачи о деформировании гибких проволочных конструкций с учетом их внутреннего строения привлекают большое внимание разработчиков нового оборудования для аэрокосмической техники, а также находят широкое применение в области энергетики, оптоволоконной техники, подводного оборудования и строительства [1-19]. О возрастающем интересе к этому направлению можно судить, например, по материалам международного симпозиума International Symposium on Cable Dynamics, который регулярно проводится на базе ведущих научно-исследовательских, промышленных и учебных организаций $[6,18]$.

В расчетной практике проволочные конструкции, подобные проводам ЛЭП, рассматриваются как однородные по структуре стержни или нити с осредненными жесткостными параметрами без учета их внутреннего строения. Такой подход используется, например, при решении задач о стрелах провеса проводов между опорами в различных эксплуатационных режимах или задач о колебаниях проводов под воздействием ветровых потоков [16]. Однако такие подходы не описывают известные эффекты при деформировании проводов [4-12], и их использование в расчетах может приводить к недопустимым ошибкам [8].

Например, для расчета изгибной и крутильной жесткостей проводов в инженерной практике используются два предположения о совместном деформировании проволочных спиралей. Они «полярны» по смыслу, определяя нижнюю и верхнюю границы теоретичес- 
кой оценки. Одно из них основывается на предположении, что проволоки деформируются независимо друг от друга. Другое - на гипотезе связанного деформирования, когда проволочные спирали мысленно жестко соединяются в одно целое, образуя своеобразный монолитный стержень. Однако сравнительные расчеты показывают [19], что величины жесткостей, посчитанные по этим методикам для выбранного провода или троса, могут различаться друг от друга более чем в 70 раз. Неопределенность в знании параметров жесткости и демпфирования проводов и других аналогичных систем приводит к недопустимым ошибкам и делает малоэффективными существующие расчетные методы и алгоритмы. Ошибочные физико-механические представления о деформировании проводов и подобных систем разрушают целостность математической модели, правильной во многих других аспектах.

Возможно использование метода «детального» конечно-элементного моделирования проволочной конструкции $[13,14]$. Однако такой путь крайне трудоемок и «субъективен», поскольку результаты расчетов в значительной степени зависят от модели и геометрии контактных поверхностей, формируемых расчетчиком. Этот подход практически неприемлем при исследовании динамического поведения проволочных конструкций или оптимизации их конструкций.

Существуют подходы, основанные на идеологии осреднения механических свойств проволочных спиралей в каждом повиве (проволочном слое) провода или троса. Например, в работах [4, 5, 9-12, 15-17] рассматривается деформирование произвольной спиральной проволоки, затем вводится гипотеза о совместной работе всех проволок повива или всего провода, которая приводит к взаимной увязке проволок в единый ансамбль. Однако в этом случае затруднительно ввести корректную модель межповивного взаимодействия с учетом сил давления и трения между соседними повивами.

Другой способ, предложенный В.И. Шалашилиным и А.Н. Данилиным и развитый в работах $[18,20-21]$, заключается в энергетическом осреднении и последующем сведении повива к некоторой эквивалентной по упругим свойствам анизотропной цилиндрической оболочке. Провод при таком способе рассматривается как система вложенных друг в друга цилиндрических оболочек, взаимодействующих между собой силами давления и трения. Отличительной чертой предлагаемого подхода является простота формулировки, наглядность, возможность получения аналитических решений, формул и эффективных в вычислительном плане алгоритмов.

В настоящее время появился класс задач, связанных с проектированием и изготовлением зажимов спирального типа, предназначенных для подвески, натяжения, соединения, защиты и ремонта проводов и тросов ЛЭП [21, 23-30]. Спиральный зажим представляет собой один или несколько повивов ограниченной длины, каждый из которых образован из отдельных спиральных проволок или их прядей. Конструкция спирального зажима очень хорошо сочетается с проводами, так как она обладает гибкостью и после монтажа фактически интегрируется с проводом в одно целое. С точки зрения механики повивы спирального зажима, смонтированного на проводе, можно рассматривать как дополнительные внешние (для провода) слои ограниченной длины.

При проектировании зажима ставится задача об определении его несущей способности, а также нахождении оптимальных значений конструктивных параметров, например длины зажима, направления и шага (угла подъема) спиралей. При неудачном их выборе работа зажима может оказаться неэффективной и даже приводить к повреждениям ядра 
конструкции. Решить эти задачи можно только на основе рассмотрения спиральной конструкций как системы взаимодействующих проволочных повивов силами давления и трения.

Научных работ, посвященных исследованию деформаций и несущей способности спиральных зажимов, немного. Экспериментальные исследования здесь имеют первостепенное значение, но вряд ли ими можно пользоваться для анализа работоспособности зажимов и оптимизации их конструкции. Наиболее интересными представляются исследования С.В. Рыжова, который предложил оригинальную методику расчета зажимов, основанную на анализе деформирования отдельной спирали, намотанной на сердечник [23, 24]. Автором при определенных допущениях построена феноменологическая модель деформирования одноповивного спирального зажима.

\section{1. Основные дифференциальные соотношения для винтового стержня}

Рассмотрим равновесие элемента винтового стержня в соприкасающейся плоскости, как показано на рис. 1. Пусть $d s-$ длина элемента, $\rho=r / \cos ^{2} \alpha$ - радиус кривизны, где $\alpha-$ угол подъема; $d \theta=d s / \rho$.

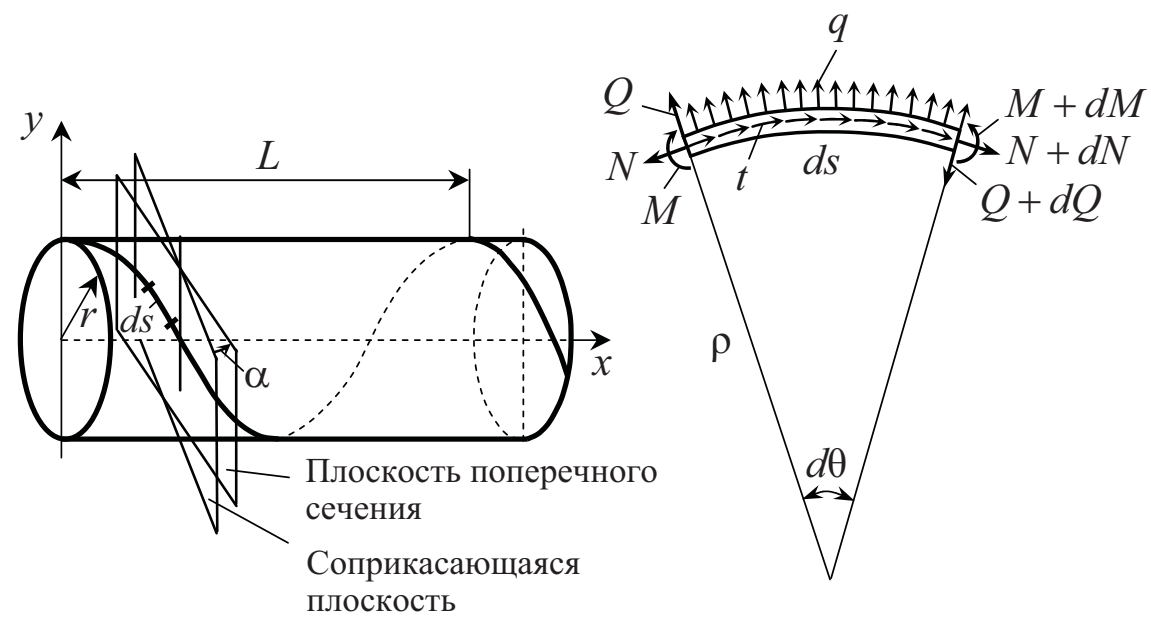

Рис. 1. Равновесие дифференциального элемента винтового стержня в соприкасающейся плоскости

Тогда уравнения равновесия сил в проекциях на ось и нормаль к оси стержня имеют вид

$$
\frac{d N}{d s}-\frac{Q}{\rho}+t=0, \quad \frac{d Q}{d s}+\frac{N}{\rho}-q=0 .
$$

Условие равенства моментов дает также известное соотношение $d M / d s=Q$.

\section{2. Сведение повива к эквивалентной цилиндрической оболочке}

Пусть $S$ - длина витка винтового стержня по его оси, а $L$ - длина шага витка по оси провода $x$. Их величины определяются формулами

$$
S=2 \pi r / \cos \alpha, L=S|\sin \alpha|=2 \pi r|\sin \alpha| / \cos \alpha .
$$


Рассмотрим деформацию витка винтового стержня под действием усилий, которые вызывают появление в стержне внутренних сил, постоянных по его длине. Сначала нагрузим стержень силой $P$ вдоль оси провода, как это показано на рис. $2, a$.

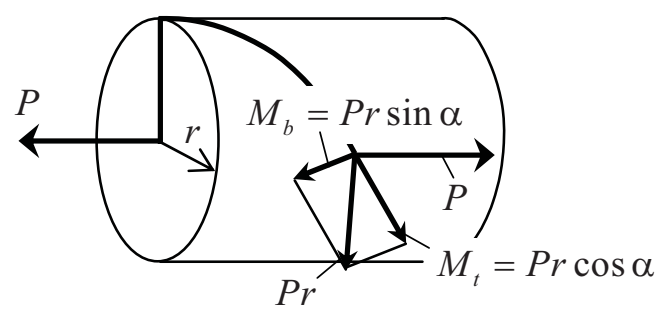

$a$

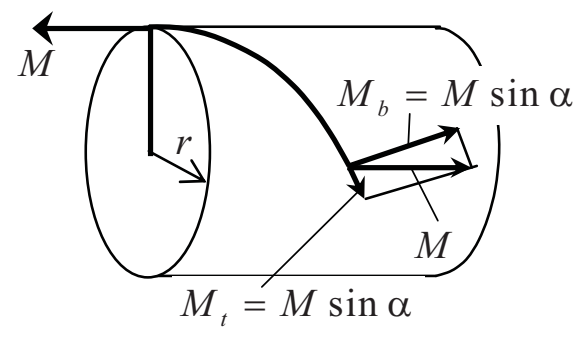

$\sigma$

Рис. 2. Моменты в сечении винтового стержня от действия внешних силовых факторов: $a$-моменты от действия продольной силы; $\sigma$ - моменты от действия крутящего момента

Вызываемый момент $\operatorname{Pr}$ в сечении стержня можно разложить на изгибающий $M_{b}(P)$ и крутящий $M_{t}(P)$ моменты. Тогда

$$
M_{b}(P)=\operatorname{Pr} \sin \alpha, M_{t}(P)=\operatorname{Pr} \cos \alpha .
$$

В сечении проволоки также появляется продольная сила

$$
N(P)=P \sin \alpha .
$$

Влияние перерезывающих сил на деформацию стержня учитывать не будем.

Рассмотрим теперь нагружение винтового стержня крутящим моментом - моментом $M$ в плоскости, нормальной к оси $x$. Как видно из рис. 2,6 , в сечениях стержня возникают следующие изгибающий и крутящий моменты:

$$
M_{b}(M)=-M \cos \alpha, M_{t}(M)=M \sin \alpha .
$$

Будем считать, что при действии поперечной нагрузки $q$ (в направлении радиуса кривизны стержня) точки оси стержня перемещаются на величину смещения $w$ строго вдоль радиусов кривизны и эти перемещения постоянны по длине стержня. Тогда, как следует из второго уравнения (1), в сечениях стержня возникает только продольная сила

$$
N(q)=q \rho=q r / \cos ^{2} \alpha .
$$

При одновременном действии всех трех нагрузок в сечениях стержня появляются суммарные изгибающие, крутящие и продольные силовые факторы, величины которых, в соответствии с формулами (3)-(6) соответственно:

$$
\begin{gathered}
M_{b}=M_{b}(P)+M_{b}(M)=P r \sin \alpha-M \cos \alpha, \\
M_{t}=M_{t}(P)+M_{t}(M)=P r \cos \alpha+M \sin \alpha, \\
N=N(P)+N(q)=P \sin \alpha+q r / \cos ^{2} \alpha .
\end{gathered}
$$

Потенциальная энергия деформации витка подсчитывается по формуле

$$
U=\frac{1}{2} \int_{0}^{S}\left(\frac{M_{b}^{2}}{E J_{z}}+\frac{M_{t}^{2}}{G J_{t}}+\frac{N^{2}}{E F}\right) d s,
$$


где $E J_{z}, G J_{t}, E F$ - жесткости на изгиб, кручение и растяжение винтового стержня соответственно направлениям моментов $M_{b}, M_{t}$ и силы $N$. После подстановки в это выражение соотношений (7) с учетом (2) получим

$$
U=\frac{1}{2} S\left[\frac{(P r \sin \alpha-M \cos \alpha)^{2}}{E J_{z}}+\frac{(P r \cos \alpha+M \sin \alpha)^{2}}{G J_{t}}+\frac{\left(P \sin \alpha+q r / \cos ^{2} \alpha\right)^{2}}{E F}\right]
$$

Выберем в качестве обобщенных перемещений удлинение $\Delta$ винтового стержня по оси $x$, угол закручивания стержня вокруг оси $x$ и площадь $S w$, где $w$ - перемещения точек витого стержня вдоль радиуса кривизны.

Введем обобщенные силы как силы, совершающие работу на этих обобщенных перемещениях. Они равны соответственно

$$
\begin{gathered}
P^{*}=P+N(q) \sin \alpha=P+q r \sin \alpha / \cos ^{2} \alpha, \\
M^{*}=M+N(q) r \cos \alpha=M+q r^{2} / \cos \alpha .
\end{gathered}
$$

Обобщенному перемещению $S w$ соответствует интенсивность $q$ усилия, действующего на стержень вдоль радиуса кривизны. Действительно, работа усилия на перемещении $w$ определяется формулой

$$
A_{q}=\int_{0}^{S} w q d s
$$

Если считать, что $w$ в пределах витка длиной $S$ является величиной постоянной, то $A_{q}=q \cdot S w$, что и определяет искомую обобщенную пару «сила-перемещение».

Подставляя в (8) выражения для $P$ и $M$ через $P^{*}, M^{*}$ и $q$, вытекающие из (9), получим

$$
\begin{gathered}
U=\frac{1}{2} S\left\{\frac{1}{E J_{z}}\left[P^{*} r \sin \alpha-M^{*} \cos \alpha-q r^{2}\left(\operatorname{tg}^{2} \alpha-1\right)\right]^{2}+\right. \\
\left.+\frac{1}{G J_{t}}\left(P^{*} r \cos \alpha+M^{*} \sin \alpha-2 q r^{2} \operatorname{tg} \alpha\right)^{2}+\frac{1}{E F}\left(P^{*} \sin \alpha+q r\right)^{2}\right\} .
\end{gathered}
$$

В соответствии с теоремой Кастильяно обобщенные перемещения определяются выражениями: $\Delta=d U / d P^{*}, \varphi=d U / d M^{*}, S w=d U / d q$. В матричной форме

$$
\left[\begin{array}{lll}
\Delta & \varphi & S w
\end{array}\right]^{T}=A\left[\begin{array}{lll}
P^{*} & M^{*} & q
\end{array}\right]^{T},
$$

где элементы матрицы $A=\left\|a_{i j}\right\|(i, j=1,2,3)$.

$$
\begin{aligned}
& a_{11}=S r^{2}\left(\frac{1+\psi}{E J_{z}} \sin ^{2} \alpha+\frac{\cos ^{2} \alpha}{G J_{t}}\right), a_{12}=a_{21}=S r\left(\frac{1}{G J_{t}}-\frac{1}{E J_{z}}\right) \sin \alpha \cos \alpha, \\
& a_{22}=S\left(\frac{\sin ^{2} \alpha}{G J_{t}}+\frac{\cos ^{2} \alpha}{E J_{z}}\right), a_{13}=a_{31}=-S r^{3}\left(\frac{\operatorname{tg}^{2} \alpha-(1+\psi)}{E J_{z}}+\frac{2}{G J_{t}}\right) \sin \alpha,
\end{aligned}
$$




$$
a_{23}=a_{32}=-S r^{2}\left(\frac{2 \operatorname{tg}^{2} \alpha}{G J_{t}}+\frac{1-\operatorname{tg}^{2} \alpha}{E J_{z}}\right) \cos \alpha, a_{33}=S r^{4}\left[\frac{\left(1-\operatorname{tg}^{2} \alpha\right)^{2}+\psi}{E J_{z}}+\frac{4 \operatorname{tg}^{2} \alpha}{G J_{t}}\right],
$$

безразмерный коэффициент $\psi=J_{z} / F r^{2}$.

Теперь необходимо рассмотреть систему из $n$ винтовых стержней (проволок) и сопоставить ее с цилиндрической оболочкой радиусом $r$ и длиной $L$, нагруженной по торцам погонным продольным усилием $T$, крутящим моментом $H$ и внутренним давлением $p$ (рис. 3).

Нагрузки $P_{1}, M_{1}, q_{1}$, действующие на отдельный стержень системы, и нагрузки, действующие на оболочку, связаны соотношениями

$$
P_{1}=T 2 \pi r / n, M_{1}=H / n, q_{1}=p 2 \pi r L /(n S),
$$

где $n$ - количество стержней.
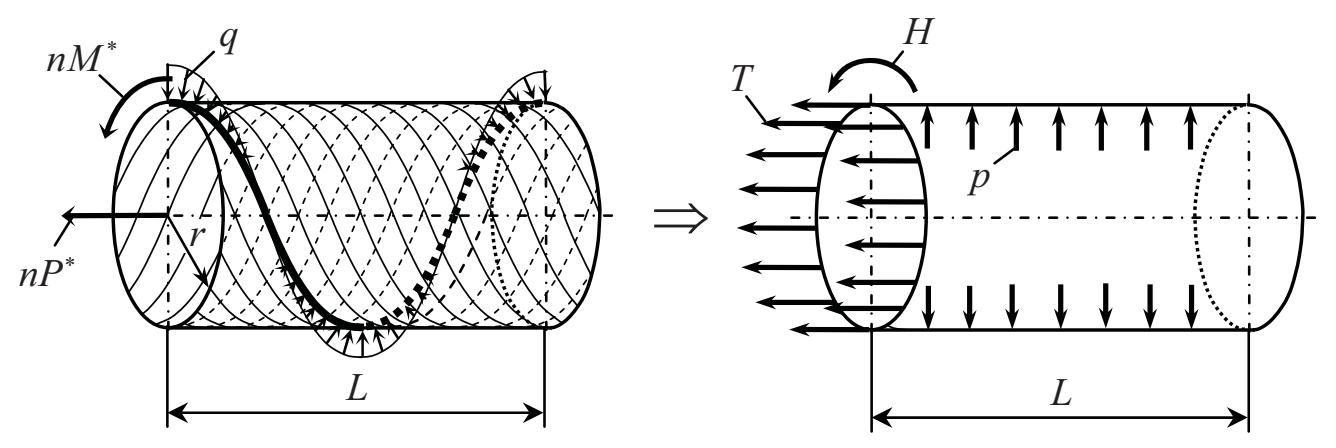

Рис. 3. Сведение одного повива провода к эквивалентной оболочке

Если ввести относительное удлинение $\varepsilon=\Delta / L$ и относительный угол закручивания $\theta=\varphi / L$, то для цилиндрической оболочки с учетом (12) получим

$$
\left[\begin{array}{lll}
\varepsilon & \theta & w
\end{array}\right]^{T}=B\left[\begin{array}{lll}
T & H & p
\end{array}\right]^{T},
$$

где элементы матрицы $B=\left\|b_{i j}\right\|(i, j=1,2,3)$ равны соответственно

$$
\begin{gathered}
b_{11}=\frac{2 \pi r^{3}}{n|\sin \alpha|}\left(\frac{1+\psi}{E J_{z}} \sin ^{2} \alpha^{(i)}+\frac{\cos ^{2} \alpha}{G J_{t}}\right), b_{12}=\frac{r}{n} \operatorname{sign} \alpha \cos \alpha\left(\frac{1}{G J_{t}}-\frac{1}{E J_{z}}\right), \\
b_{13}=-\frac{2 \pi r^{4}}{n} \sin \alpha\left(\frac{\operatorname{tg}^{2} \alpha-1-\psi}{E J_{z}}+\frac{2}{G J_{t}}\right) \\
b_{21}=2 \pi r b_{12}, b_{22}=\frac{1}{n|\sin \alpha|}\left(\frac{\sin ^{2} \alpha}{G J_{t}}+\frac{\cos ^{2} \alpha}{E J_{z}}\right), \\
b_{23}=-\frac{2 \pi r^{3}}{n} \cos \alpha\left(\frac{2 \operatorname{tg}^{2} \alpha}{G J_{t}}+\frac{1-\operatorname{tg}^{2} \alpha}{E J_{z}}\right) \\
b_{31}=b_{13}, b_{32}=\frac{b_{23}}{2 \pi r}, b_{33}=\frac{2 \pi r^{5}}{n}|\sin \alpha|\left[\frac{\left(1-\operatorname{tg}^{2} \alpha\right)^{2}+\psi}{E J_{z}}+\frac{4 \operatorname{tg}^{2} \alpha}{G J_{t}}\right] .
\end{gathered}
$$

\section{3. О жесткостях провода}


Будем трактовать провод как стержень со специфичными механическими свойствами. Это позволяет записать соотношения между усилиями, действующими на стержень, и его деформациями в пределах закона Гука в общем виде:

$$
\left(\begin{array}{lll}
\varepsilon & \theta & \kappa
\end{array}\right)^{T}=B\left(\begin{array}{lll}
N H & M
\end{array}\right)^{T} \text { или }\left(\begin{array}{lll}
N H & M
\end{array}\right)^{T}=R\left(\begin{array}{lll}
\varepsilon & \theta & \kappa
\end{array}\right)^{T} .
$$

Здесь $\varepsilon, \theta, \kappa-$ относительное удлинение, относительный угол закручивания и кривизна оси стержня; $N, H, M$ - продольная сила, крутящий и изгибающий моменты в стержне; $B$ и $R$ - матрицы податливостей и жесткостей, связанные соотношением $R=B^{-1}$.

Традиционное определение жесткостей на растяжение, кручение и изгиб следует из (15), если матрицы $B$ и $R$ диагональны. Этому соответствует случай, когда рассматривается изотропный стержень в главных центральных осях.

Провод представляет собой систему, состоящую из сердечника (центральной проволоки) и проволочных слоев (повивов), каждый из которых можно моделировать как анизотропную цилиндрическую безмоментную оболочку с соотношениями вида (13), (14).

Будем считать, что слои провода уложены без зазора и натяга. Пронумеруем слои от 1 до $n$. Центральному проводу присвоим индекс (0). Тогда для $i$-го повива соотношение (13) примет вид

$$
\left(\begin{array}{lll}
\varepsilon^{(i)} & \theta^{(i)} & 0
\end{array}\right)^{T}=B^{(i)}\left(T^{(i)} H^{(i)} p^{(i)}\right)^{T} .
$$

Здесь $T^{(i)}$ - продольное усилие в срединной поверхности оболочки, которое связано с силой $N^{(i)}$, действующей на оболочку, соотношением

$$
N^{(i)}=2 \pi r^{(i)} T^{(i)}
$$

где $r^{(i)}$ - радиус срединной поверхности $i$-го повива.

После исключения $p^{(i)}$ из соотношений (16), последние сводятся к виду

$$
\left(\varepsilon^{(i)} \theta^{(i)}\right)^{T}=\bar{B}^{(i)}\left(T^{(i)} H^{(i)}\right)^{T},
$$

где матрица

$$
\bar{B}^{(i)}=\left\|\bar{b}_{k l}\right\|=\left(\begin{array}{ll}
b_{11}^{(i)}-\frac{b_{13}^{(i)} b_{31}^{(i)}}{b_{33}^{(i)}} & b_{12}^{(i)}-\frac{b_{13}^{(i)} b_{32}^{(i)}}{b_{33}^{(i)}} \\
b_{21}^{(i)}-\frac{b_{23}^{(i)} b_{31}^{(i)}}{b_{33}^{(i)}} & b_{22}^{(i)}-\frac{b_{23}^{(i)} b_{32}^{(i)}}{b_{33}^{(i)}}
\end{array}\right) ; k, l=1,2 .
$$

Отсюда

$$
\left(T^{(i)} H^{(i)}\right)^{T}=C^{(i)}\left(\varepsilon^{(i)} \theta^{(i)}\right)^{T}, C^{(i)}=\left\|c_{p q}^{(i)}\right\|=\left(\bar{B}^{(i)}\right)^{-1} ; p, q=1,2 .
$$

При рассмотрении провода в целом возникающие в нем продольная сила $N$ и крутящий момент $H$ складываются из сил и моментов, действующих на сердечник и повивы. Поэтому 


$$
N=N^{(0)}+\sum_{i=1}^{n} N^{(i)}=N^{(0)}+2 \pi \sum_{i=1}^{n} r^{(i)} T^{(i)}, H=H^{(0)}+\sum_{i=1}^{n} H^{(i)} .
$$

Будем считать, что повивы провода не проскальзывают друг относительно друга, т.е. их деформации одинаковы. Тогда

$$
\varepsilon^{(i)}=\varepsilon, \quad \theta^{(i)}=\theta ; \quad i=0,1, \ldots, n .
$$

Соотношения (21) можно представить в матричной форме:

$$
\left(\begin{array}{l}
N \\
H
\end{array}\right)=\left(\begin{array}{l}
N^{(0)} \\
H^{(0)}
\end{array}\right)+\sum_{i=1}^{n}\left(\begin{array}{cc}
2 \pi r^{(i)} & 0 \\
0 & 1
\end{array}\right)\left(\begin{array}{l}
T^{(i)} \\
H^{(i)}
\end{array}\right) .
$$

Подставляя сюда (20) с учетом (22), получим

$$
\left(\begin{array}{l}
N \\
H
\end{array}\right)^{T}=R\left(\begin{array}{l}
\varepsilon \\
\theta
\end{array}\right)^{T}, R=\left(\begin{array}{cc}
E^{(0)} F^{(0)} & 0 \\
0 & G^{(0)} J_{t}^{(0)}
\end{array}\right)+\sum_{i=1}^{n}\left(\begin{array}{cc}
2 \pi r^{(i)} & 0 \\
0 & 1
\end{array}\right) C^{(i)} .
$$

Здесь $E^{(0)}, G^{(0)}$ - модули на растяжение и сдвиг сердечника; площадь $F^{(0)}=\pi d^{(0) 2} / 4$, полярный момент инерции $J_{t}^{(0)}=\pi d^{(0) 4} / 32 ; d^{(0)}$ - диаметр проволоки сердечника.

Полученная в результате матрица $R$ является матрицей жесткости для провода при его работе на растяжение и кручение. Следует ожидать, что ввиду встречной намотки повивов провода элементы $R_{12}=R_{21}$ малы по сравнению с диагональными $R_{11}$ и $R_{22}$. Во всяком случае углы намотки повивов могут быть подобраны так, чтобы недиагональные элементы оказались величинами малыми. Тогда $R_{11}$ и $R_{22}$ можно рассматривать как классические жесткости провода на растяжение и кручение.

Соотношения (18), (19) позволяют определить жесткости проволочной конструкции на кручение и изгиб.

Рассмотрим сначала кручение. При $T=0$ из (18) следует, что для $i$-го повива

$$
\theta^{(i)}=\bar{b}_{22}^{(i)} H^{(i)} .
$$

При закручивании провода на угол $\theta$ все повивы закручиваются одинаково:

$$
\theta^{(i)}=\theta ; i=0,1, \ldots, n \text {. }
$$

В то же время крутящий момент $H$ складывается из крутящих моментов составляющих повивов:

$$
H=\sum_{i=0}^{n} H^{(i)}
$$

Поэтому, учитывая (23)-(25), имеем

$$
H=\sum_{i=0}^{n} \theta^{(i)} c_{22}^{(i)}=\theta \sum_{i=0}^{n} c_{22}^{(i)}
$$

Обозначим жесткость провода на кручение через $G J_{t}$, где $G-$ некоторый условный модуль сдвига. Тогда 


$$
\theta=\frac{H}{G J_{t}}
$$

Сравнивая выражения (26) и (27), получим

$$
G J_{t}=\sum_{i=0}^{n} c_{22}^{(i)}
$$

Для сердечника и $i$-го повива

$$
\begin{gathered}
b_{22}^{(0)}=\frac{1}{G^{(0)} J_{t}^{(0)}} \approx \frac{10}{G^{(0)} d^{(0) 4}} \\
J_{t}^{(i)}=0,1 d^{(i) 4}, J_{b}^{(i)}=0,05 d^{(i) 4}, G^{(i)}=\frac{E^{(i)}}{2\left(1+\mu^{(i)}\right)},
\end{gathered}
$$

где $E^{(i)}, \mu^{(i)}$ - модуль упругости и коэффициент Пуассона материала $i$-го повива. С учетом последних формул выражение (28) преобразуется к виду

$$
G J_{t}=0,1 G^{(0)} J_{t}^{(0)}+\sum_{i=1}^{n} c_{22}^{(i)}
$$

Рассмотрим изгибную жесткость провода. Кривизна к, радиус кривизны $\rho$ и изгибающий момент $M_{b}$ стержня связаны соотношением

$$
\kappa=1 / \rho=M_{b} / E J_{b} .
$$

Момент $M_{b}$, подсчитанный через нормальные напряжения $\sigma$ в поперечном сечении $F$,

$$
M_{b}=\int_{F} y \sigma d F,
$$

где $y$ - расстояние от элемента $d F$ до нейтрального волокна.

При выполнении гипотезы плоских сечений удлинение волокна стержня с сечением $d F$

$$
\varepsilon=y / \rho .
$$

Если рассматривать $i$-й повив как оболочку, состоящую из продольных волокон, в которых действуют напряжения $\sigma^{(i)}=T^{(i)} / d^{(i)}$, то из (18), (19) следует закон Гука для продольного волокна в виде $\varepsilon^{(i)}=\bar{b}_{11}^{(i)} T^{(i)}=\bar{b}_{11}^{(i)} d^{(i)} \sigma^{(i)}$. Тогда для $i$-го повива из (30)-(32) получаем

$$
E^{(i)} J_{b}^{(i)}=M_{b}^{(i)} \rho^{(i)}=\rho \int_{F} y \frac{\varepsilon^{(i)}}{\bar{b}_{11}^{(i)} d^{(i)}} d F=\frac{1}{\bar{b}_{11}^{(i)} d^{(i)}} \int_{F^{(i)}} y^{2} d F^{(i)} .
$$

Для кольцевого сечения радиусом $r^{(i)}$ и толщиной $d^{(i)}$ интеграл $\int_{F^{(i)}} y^{2} d F^{(i)}=\pi r^{(i) 3} d^{(i)}$. Поэтому жесткость на изгиб $i$-го повива

$$
E^{(i)} J_{b}^{(i)}=\pi r^{(i) 3} / \bar{b}_{11}^{(i)}
$$


При изгибе провода кривизна изгиба осей всех повивов и сердечника провода одинакова, а $M_{b}=\sum_{i=0}^{n} M_{b}^{(i)}$. Поэтому изгибная жесткость провода с учетом (33)

$$
E J_{b}=M_{b} \rho=\rho \sum_{i=0}^{n} M_{b}^{(i)}=\rho \sum_{i=0}^{n} \frac{E^{(i)} J_{b}^{(i)}}{\rho}=0.05 d^{(0) 4} E^{(0)}+\pi \sum_{i=1}^{n} r^{(i) 3} c_{11}^{(i)} .
$$

\section{4. Жесткости проводов серии АС}

Рассчитывались крутильные и изгибные жесткости проводов алюминиево-стальной серии российского производства $(\mathrm{AC})[1,2]$. Типовое сечение провода $\mathrm{AC}$ показано на рис. 4. Повивы 1 и 2 представляют собой токопроводящие алюминиевые повивы. Стальной

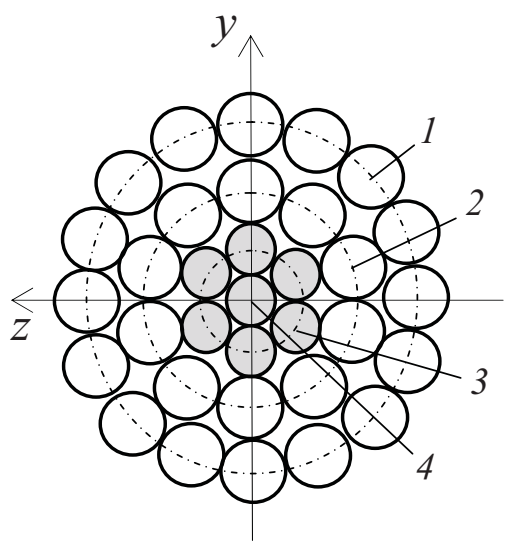

Рис. 4. Конструкция провода АС сердечник образован повивом 3 и проволокой 4 в центре. Данные для расчетов представлены в табл. 1, 2. В первых столбцах таблиц даны марки сталеалюминиевых проводов серии АС в виде отношения номинальных площадей алюминиевой и стальной частей сечения провода $\left(\mathrm{mм}^{2}\right)$. Во вторых столбцах даны в виде сумм количества проволок в каждом из повивов: значение слагаемого дает число проволок в повиве, а число слагаемых суть число повивов. В третьих и четвертых столбцах указаны номинальные диаметры проволок и кратности скрутки, определяющие углы подъема и скрутки. Кратность скрутки повива определяется формулой $m=L /(2 r+d)$, где $L-$ шаг скрутки; $r$ - радиус повива, т.е. радиус окружности, на которой лежат центры поперечных сечений проволок; $d$ - диаметр проволок повива. Из этой формулы следует, что $L=m(2 r+d)$, откуда по второй формуле (2) находим $\alpha=\operatorname{Arctg}(L / 2 \pi r)$.

Таблица 1

Параметры токопроводящей (алюминиевой) части проводов серии АС

\begin{tabular}{|c|c|c|c|}
\hline Марка провода АС & $\begin{array}{c}\text { Числа проволок } \\
\text { в повивах }\end{array}$ & $\begin{array}{c}\text { Номинальный диаметр } \\
\text { проволок, мм }\end{array}$ & $\begin{array}{c}\text { Кратности скрутки } \\
\text { повивов }\end{array}$ \\
\hline 1 & 2 & 3 & 4 \\
\hline $120 / 19$ & $10+16$ & 2,40 & 15,12 \\
\hline $120 / 27$ & $12+18$ & 2,20 & 15,12 \\
\hline $150 / 19$ & $9+15$ & 2,80 & 15,12 \\
\hline $150 / 34$ & $12+18$ & 2,50 & 15,12 \\
\hline $185 / 24$ & $9+15$ & 3,15 & 15,12 \\
\hline $240 / 39$ & $10+16$ & 3,40 & 15,12 \\
\hline $185 / 128$ & $24+30$ & 2,10 & 15,12 \\
\hline $300 / 39$ & $9+15$ & 4,00 & 15,12 \\
\hline $300 / 66$ & $12+18$ & 3,50 & 15,12 \\
\hline
\end{tabular}


Окончание табл. 1

\begin{tabular}{|c|c|c|c|}
\hline 1 & 2 & 3 & 4 \\
\hline $330 / 30$ & $10+16+22$ & 2,98 & $15,12,10$ \\
\hline $330 / 43$ & $12+18+24$ & 2,80 & $15,12,10$ \\
\hline $400 / 22$ & $10+16+22+28$ & 2,57 & $18,15,13,11$ \\
\hline $400 / 64$ & $10+16$ & 4,37 & 15,12 \\
\hline $400 / 93$ & $12+18$ & 4,15 & 15,12 \\
\hline $300 / 204$ & $24+30$ & 2,65 & 15,12 \\
\hline $500 / 26$ & $8+14+20$ & 3,90 & $15,12,10$ \\
\hline $500 / 64$ & $12+18+24$ & 3,40 & $15,12,10$ \\
\hline $550 / 71$ & $12+18+24$ & 3,60 & $15,12,10$ \\
\hline $600 / 72$ & $12+18+24$ & 3,70 & $15,12,10$ \\
\hline $650 / 79$ & $15+21+27+33$ & 2,90 & $18,15,12,10$ \\
\hline
\end{tabular}

Таблица 2

Параметры сердечников - стальной части проводов серии АС

\begin{tabular}{|c|c|c|c|}
\hline Марка провода АС & $\begin{array}{c}\text { Числа проволок } \\
\text { в повивах }\end{array}$ & $\begin{array}{c}\text { Номинальный диаметр } \\
\text { проволок, мм }\end{array}$ & $\begin{array}{c}\text { Кратности скрутки } \\
\text { повивов }\end{array}$ \\
\hline $120 / 19$ & $1+6$ & 1,85 & 20 \\
\hline $120 / 27$ & $1+6$ & 2,20 & 20 \\
\hline $150 / 19$ & $1+6$ & 1,85 & 20 \\
\hline $150 / 34$ & $1+6$ & 2,50 & 20 \\
\hline $185 / 24$ & $1+6$ & 2,10 & 20 \\
\hline $240 / 39$ & $1+6$ & 2,65 & $25,20,15$ \\
\hline $185 / 128$ & $1+6+12+18$ & 2,10 & 20 \\
\hline $300 / 39$ & $1+6$ & 2,65 & 20,20 \\
\hline $300 / 66$ & $1+6+12$ & 2,10 & 20 \\
\hline $330 / 30$ & $1+6$ & 2,30 & 20 \\
\hline $330 / 43$ & $1+6$ & 2,80 & 20 \\
\hline $400 / 22$ & $1+6$ & 2,00 & 25,20 \\
\hline $400 / 64$ & $1+6$ & 3,40 & $25,20,18$ \\
\hline $400 / 93$ & $1+6+12$ & 2,50 & 20 \\
\hline $300 / 204$ & $1+6+12+18$ & 2,65 & 20 \\
\hline $500 / 26$ & $1+6$ & 2,20 & 20 \\
\hline $500 / 64$ & $1+6$ & 3,40 & 25,20 \\
\hline $550 / 71$ & $1+6$ & 3,60 & 2,20 \\
\hline $600 / 72$ & $1+6+12$ & 2,30 & 20 \\
\hline $650 / 79$ & $1+6+12$ & & 20 \\
\hline
\end{tabular}


Результаты вычислений представлены в табл. 3. Здесь первая колонка - марка провода, вторая - внешний диаметр провода (диаметр окружности, в которую вписано поперечное сечение провода). Третья и четвертая колонки таблицы содержат значения изгибной и крутильной жесткостей, посчитанные соответственно по формулам (34) и (29). Пятая колонка содержит значения крутильных жесткостей, вычисленных по формуле $G J_{t}=0,00027 d^{4}$, где $d-$ внешний диаметр провода в миллиметрах, а числовой коэффициент имеет размерность $\mathrm{H} \cdot \mathrm{M}^{2} / \mathrm{MM}^{4}$. Эта формула получена в институте Montefiore (Бельгия, Льежский университет) в результате обширного анализа экспериментальных данных [7]. Систематических данных об изгибных жесткостях проводов, измеренных в результате каких-либо экспериментов, в литературе найти не удалось.

Сравнение 4-й и 5-й колонок табл. 3 говорит о хорошем соответствии предложенной модели экспериментальным данным.

Таблица 3

Жесткости проводов серии АС

\begin{tabular}{|c|c|c|c|c|}
\hline Марка провода АC & $d$, мм & $E J_{b}, \mathrm{H} \cdot \mathrm{m}^{2}$ & $G J_{t}, \mathrm{H} \cdot \mathrm{M}^{2}$ & 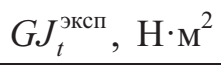 \\
\hline $120 / 19$ & 15,15 & 113,6 & 15,37 & 14,22 \\
\hline $120 / 27$ & 15,40 & 125,3 & 16,50 & 15,19 \\
\hline $150 / 19$ & 16,75 & 166,8 & 23,03 & 21,25 \\
\hline $150 / 34$ & 17,50 & 208,9 & 27,50 & 25,32 \\
\hline $185 / 24$ & 18,90 & 269,5 & 37,18 & 34,45 \\
\hline $240 / 39$ & 21,55 & 463,1 & 62,60 & 58,23 \\
\hline $185 / 128$ & 23,10 & 835,9 & 82,66 & 76,88 \\
\hline $300 / 39$ & 23,95 & 695,4 & 96,14 & 88,84 \\
\hline $300 / 66$ & 24,50 & 812,2 & 101,48 & 97,28 \\
\hline $330 / 30$ & 24,78 & 807,5 & 103,77 & 101,81 \\
\hline $330 / 43$ & 25,20 & 867,2 & 120,92 & 108,88 \\
\hline $400 / 22$ & 26,56 & 1041,6 & 163,73 & 134,36 \\
\hline $400 / 64$ & 27,68 & 1261,3 & 170,52 & 158,50 \\
\hline $400 / 93$ & 29,10 & 1615,0 & 200,79 & 193,61 \\
\hline $300 / 204$ & 29,15 & 2119,1 & 209,61 & 194,95 \\
\hline $500 / 26$ & 30,00 & 1684,2 & 244,93 & 218,70 \\
\hline $500 / 64$ & 30,60 & 1885,4 & 262,89 & 236,73 \\
\hline $550 / 71$ & 32,40 & 2369,7 & 330,42 & 297,54 \\
\hline $600 / 72$ & 33,20 & 2632,8 & 360,17 & 328,03 \\
\hline $650 / 79$ & 34,70 & 3134,4 & 468,10 & 391,45 \\
\hline
\end{tabular}

\section{5. Расчет натяжного спирального зажима}

Характерным представителем арматуры спирального типа является спиральный натяжной зажим (рис. 5), конструкция которого состоит из одного или нескольких повивов, каждый из которых образован из прядей проволоки с использованием специальных фрикционных покрытий [27-30]. Предназначен спиральный зажим для подвески и натяжения проводов, тросов и кабелей ВЛ. Его монтаж легко осуществляется вручную последовательной навивкой повивов. 


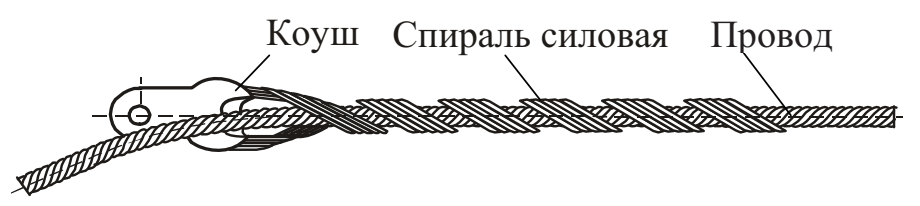

Рис. 5. Натяжной спиральный зажим на проводе

При проектировании зажима ставится задача о нахождении оптимальных значений его параметров, например длины зажима, направления и шага (угла намотки) спиралей, коэффициентов трения. В противном случае работа зажима может оказаться неэффективной и даже приводить к повреждению сердечника.

Спиральный зажим можно, так же как и повив провода, свести к эквивалентной цилиндрической оболочке.

Для решения задачи о взаимодействии зажима с проводом рассмотрим равновесие элементарного кольца длиной $d x$, эквивалентной зажиму оболочки, под действием продольного усилия $T$, окружного усилия $h=H /\left(2 \pi r^{2}\right)$ и силы трения $f$, составляющие которой в продольном и окружном направлениях обозначим через $f_{x}$ и $f_{y}$. Элемент такого кольца длиной $d y$ в окружном направлении показан на рис. 6.

Из условий равновесия элемента следуют

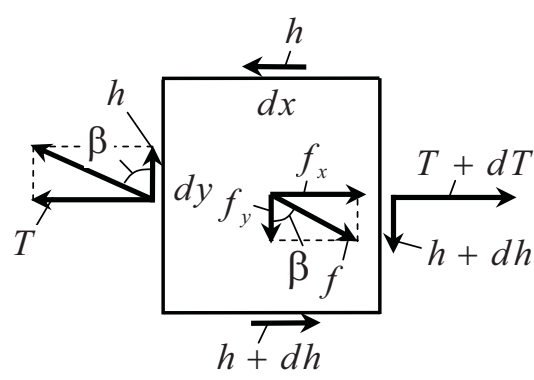

Рис. 6. Равновесие элемента кольца эквивалентной оболочки уравнения

$$
\frac{d T}{d x}=-f_{x}, \quad \frac{d H}{d x}=-2 \pi r^{2} f_{y} .
$$

Будем считать, что сила трения, распределенная по поверхности контакта между зажимом и кабелем, равна $f=k_{\mathrm{T}} p$, где $p$ - давление по поверхности контакта; $k_{\mathrm{T}}-$ коэффициент трения. Будем также считать, что сила $f$ направлена вдоль равнодействующей сдвигающего усилия, образованного силами $T$ и $h$, т.е.

$$
\begin{gathered}
f_{x}=f \sin \beta, f_{y}=f \cos \beta ; \\
\sin \beta=\frac{|T|}{\sqrt{T^{2}+h^{2}}}, \quad \cos \beta=\frac{|h|}{\sqrt{T^{2}+h^{2}}} ; \quad h=\frac{H}{2 \pi r^{2}} .
\end{gathered}
$$

Контактное усилие $p$ при заданных усилиях $T$ и $h$ и радиальном перемещении (натяге) зажима $w_{0}$ определится из третьего уравнения (13) как

$$
p=\left(w_{0}-b_{31} T-b_{32} H\right) / b_{33} .
$$

Если не учитывать уменьшение диаметра кабеля за счет его взаимодействия с зажимом, то величина $w_{0}$ равна разности начальных радиусов провода и зажима, считая ее положительной, если начальный радиус зажима меньше радиуса провода.

Если условия закрепления зажима на опоре, т.е. при $x=0$, позволяют определить начальные значения $T_{0}$ и $H_{0}$ усилия $T$ и момента $H$, то уравнения (35), (36) позволяют сформулировать начальную задачу 


$$
\begin{gathered}
\frac{d T}{d x}=-k_{\mathrm{T}} p \sin \beta, \quad \frac{d H}{d x}=-2 \pi r^{2} k_{\mathrm{T}} p \cos \beta ; \\
T(0)=T_{0}, H(0)=H_{0},
\end{gathered}
$$

где давление $p$ дается формулой (37).

Такая постановка предполагает, что длина зажима настолько велика, что взаимовлиянием его концов можно пренебречь, т.е. рассматривать зажим как полубесконечную оболочку.

В качестве первого приближения рассмотрим такое условие закрепления зажима на опоре, которое позволяет ему свободно поворачиваться, т.е. примем $H_{0}=0$. Тогда второе уравнение (38) имеет решение $H(x) \equiv 0$, а для первого уравнения начальная задача принимает вид

$$
\frac{d T}{d x}=-k_{T} \frac{w_{0}-b_{31} T}{b_{33}}=-k_{\mathrm{T}}\left(a_{0}+a_{1} T\right), T(0)=T_{0},
$$

где $a_{0}=w_{0} / b_{33}, a_{1}=-b_{31} / b_{33}$.

Ее решение выглядит так:

$$
T(x)=T_{\infty}+\left(T_{0}-T_{\infty}\right) e^{-k_{T} a_{1} x},
$$

где $T_{\infty}=-a_{0} / a_{1}$.

Отметим, что решение (39) имеет асимптотическую составляющую $T_{\infty}=\lim _{x \rightarrow \infty} T=-w_{0} /\left|b_{31}\right|$. Характер его изменения показан на рис. 7. Из него видно, что длина зажима, необходимая для того, чтобы он удерживал усилие $T_{0}$, должна быть не меньше $l_{\min }$. Из решения (39) следует, что величина $l_{\min }$

$$
l_{\min }=\frac{1}{k_{\mathrm{T}} a_{1}} \ln \left(1-\frac{T_{0}}{T_{\infty}}\right) .
$$

Рассмотрим теперь задачу взаимодействия в системе, показанной на рис. 8 , состоящей из зажима, внешнего повива провода и внутренней его части, которую будем называть ядром.

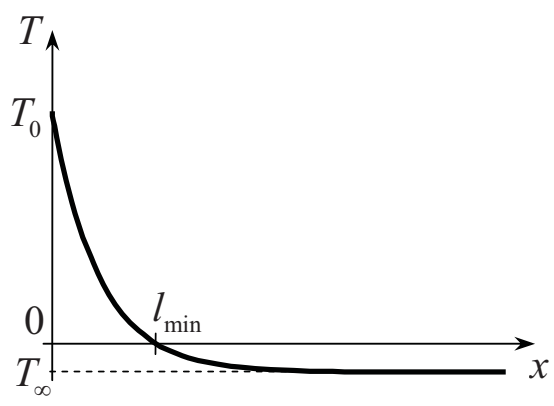

Рис. 7. Характер изменения решения (39)

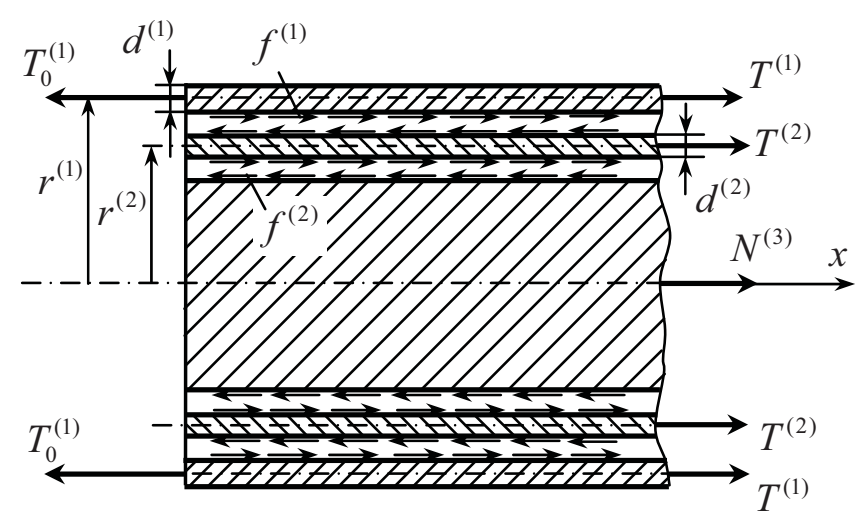

Рис. 8. Взаимодействие зажима, внешнего повива провода и внутренней его части 
Уравнения равновесия зажима и внешнего повива провода имеют вид

$$
\begin{gathered}
2 \pi r^{(1)} \frac{d T^{(1)}}{d x}=-f_{x}^{(1)} 2 \pi\left(r^{(1)}-\frac{d^{(1)}}{2}\right), \\
\frac{d H^{(1)}}{d x}=-f_{y}^{(1)} 2 \pi\left(r^{(1)}-\frac{d^{(1)}}{2}\right)^{2}, \\
2 \pi r^{(2)} \frac{d T^{(2)}}{d x}=f_{x}^{(1)} 2 \pi\left(r^{(2)}+\frac{d^{(2)}}{2}\right)-f_{x}^{(2)} 2 \pi\left(r^{(2)}-\frac{d^{(2)}}{2}\right), \\
\frac{d H^{(2)}}{d x}=f_{y}^{(1)} 2 \pi\left(r^{(2)}+\frac{d^{(2)}}{2}\right)^{2}-f_{y}^{(2)} 2 \pi\left(r^{(2)}-\frac{d^{(2)}}{2}\right)^{2},
\end{gathered}
$$

а усилие $N^{(3)}$ в ядре провода определяется из условия равновесия в форме

$$
N^{(3)}+T^{(1)} 2 \pi r^{(1)}+T^{(2)} 2 \pi r^{(2)}=T_{0}^{(1)} 2 \pi r^{(1)} .
$$

Если считать, что возникающие между слоями силы трения $f^{(1)}$ и $f^{(2)}$ пропорциональны силам давления между слоями $p^{(1)}$ и $p^{(2)}$, то

$$
f^{(1)}=k_{T}^{(1)} p^{(1)}, f^{(2)}=k_{T}^{(2)} p^{(2)} .
$$

Как и ранее, будем предполагать, что силы трения направлены вдоль равнодействующей сдвигающего усилия между слоями. Тогда

$$
\begin{gathered}
f_{x}^{(1)}=f^{(1)} \sin \beta^{(1)}, f_{y}^{(1)}=f^{(1)} \cos \beta^{(1)} \\
\cos \beta^{(1)}=\left|h^{(1)}\right| / \sqrt{T^{(1)^{2}}+h^{(1)^{2}}}, \sin \beta^{(1)}=\left|T^{(1)}\right| / \sqrt{T^{(1)^{2}}+h^{(1)^{2}}}, \\
h^{(1)}=H^{(1)} /\left(2 \pi r^{(1)^{2}}\right), \\
f_{x}^{(2)}=f^{(2)} \sin \beta^{(2)}, f_{y}^{(2)}=f^{(2)} \cos \beta^{(2)} \\
\cos \beta^{(2)}=\left|h_{12}\right| / \sqrt{T_{12}^{2}+h_{12}^{2}}, \sin \beta^{(2)}=\left|T_{12}\right| / \sqrt{T_{12}^{2}+h_{12}^{2}} \\
h^{(2)}=H^{(2)} /\left(2 \pi r^{(2)^{2}}\right), \\
T_{12}=T^{(1)}\left(r^{(1)} / r^{(2)}\right)+T^{(2)}, h_{12}=h^{(1)}\left(r^{(1)} / r^{(2)}\right)+h^{(2)} .
\end{gathered}
$$

Давление $p^{(1)}$ между зажимом и проводом определяется соотношением (37):

$$
p^{(1)}=\left(w_{0}^{(1)}-b_{31}^{(1)} T^{(1)}-b_{32}^{(1)} H^{(1)}\right) / b_{33}^{(1)} \text {; }
$$

давление $p^{(2)}$ между внешним повивом провода и его ядром определяется тем же соотношением, но записанным для внешнего повива, принимая его прогиб равным нулю:

$$
p^{(2)}=p^{(1)}\left(r^{(1)} / r^{(2)}\right)-\left(b_{31}^{(2)} T^{(2)}+b_{32}^{(2)} H^{(2)}\right) / b_{33}^{(2)} .
$$


Как и ранее, будем считать, что зажим на опоре может свободно поворачиваться. Тогда для уравнений (40) с учетом обозначений (41)-(44) можно сформулировать следующую начальную задачу:

$$
\begin{gathered}
\frac{d T^{(1)}}{d x}=-f_{x}^{(1)}\left(1-\frac{d^{(1)}}{2 r^{(1)}}\right), \\
\frac{d H^{(1)}}{d x}=-f_{y}^{(1)} 2 \pi r^{(1)}\left(1-\frac{d^{(1)}}{2 r^{(1)}}\right)^{2}, \\
\frac{d T^{(2)}}{d x}=f_{x}^{(1)}\left(1+\frac{d^{(2)}}{2 r^{(2)}}\right)-f_{x}^{(2)}\left(1-\frac{d^{(2)}}{2 r^{(2)}}\right), \\
\frac{d H^{(2)}}{d x}=f_{y}^{(1)} 2 \pi r^{(2)}\left(1+\frac{d^{(2)}}{2 r^{(2)}}\right)^{2}-f_{y}^{(2)} 2 \pi r^{(2)}\left(1-\frac{d^{(2)}}{2 r^{(2)}}\right)^{2} ; \\
f^{(1)}=k_{T}^{(1)} p^{(1)}, f^{(2)}=k_{T}^{(2)} p^{(2)} ; \\
\left.f_{x}^{(1)}=f_{0}^{(1)}-b_{31}^{(1)} T^{(1)}-b_{32}^{(1)} H^{(1)}\right) / b_{33}^{(1)}, p^{(2)}=p^{(1)}\left(r^{(1)} / r^{(2)}\right)-\left(b_{31}^{(2)} T^{(2)}+b_{32}^{(2)} H^{(2)}\right) / b_{33}^{(2)} ; \\
T^{(1)}(0)=f_{0}^{(1)} \cos \beta^{(1)}, f_{x}^{(2)}=f^{(2)} \sin \beta^{(2)}, f_{y}^{(2)}=f^{(2)} \cos \beta^{(2)} ;
\end{gathered}
$$

В случае жесткого закрепления зажима на опоре, когда $\theta^{(1)}=0$, из второго уравнения (13) с учетом выражения для $p^{(1)}(43)$ следует, что

$$
H_{0}^{(1)}=-\left(b_{22}^{(1)}-b_{23}^{(1)} \frac{b_{32}^{(1)}}{b_{33}^{(1)}}\right)^{-1}\left[\frac{b_{23}^{(1)}}{b_{33}^{(1)}} w_{0}^{(1)}+\left(b_{21}^{(1)}-b_{23}^{(1)} \frac{b_{31}^{(1)}}{b_{33}^{(1)}}\right) T_{0}^{(1)}\right]
$$

Тогда начальные условия для уравнений (45) запишутся в виде

$$
T^{(1)}(0)=T_{0}^{(1)}, T^{(2)}(0)=0, H^{(1)}(0)=H_{0}^{(1)}, H^{(2)}(0)=0,
$$

где $H_{0}^{(1)}$ определяется формулой (47).

В качестве примера рассмотрим задачу о взаимодействии натяжного зажима марки НС-17,1-01 (по каталогу ЭССП от ХІ. 2004 г.) и провода марки АС 150/24 [27]. Параметры зажима: радиус срединной поверхности $r^{(1)}=10,45 \mathrm{MM}$; диаметр проволок $d^{(1)}=3,8 \mathrm{Mм} ;$ модуль на растяжение $E^{(1)}=20000 \kappa \Gamma / \mathrm{Mм}^{2} ;$ коэффициент Пуассона $\mu^{(1)}=0,29$; угол наклона проволок относительно поперечного сечения провода $\alpha^{(1)}=67^{\circ}$; количество проволок $n^{(1)}=14$; технологический натяг $w_{0}^{(1)}=0,9$ мм. Коэффициент трения между анизотропными оболочками, моделирующими повивы зажима и провода, принимался $k_{\mathrm{T}}^{(1)}=0,6$.

Параметры внешнего повива провода: радиус срединной поверхности $r^{(2)}=7,2$ мм; диаметр проволок $d^{(2)}=2,7 \mathrm{Mм}$; модуль на растяжение $E^{(2)}=6300 \kappa \Gamma / \mathrm{Mм}^{2} ;$ коэффициент Пуассона $\mu^{(2)}=0,34 ;$ угол наклона проволок относительно поперечного сечения провода 
$\alpha^{(2)}=103,6^{\circ}$; количество проволок $n^{(2)}=16$; технологический натяг $w_{0}^{(2)}=0,2$ мм. Коэффициент трения между оболочкой, моделирующей внешний повив провода, и цилиндрической поверхностью ядра провода (внутренней части провода) принимался равным $k_{\mathrm{T}}^{(2)}=0,15$.

На графиках рис. 9 представлены соответственно зависимости усилий $T^{(i)}$ и крутящих моментов $H^{(i)}$, действующих в зажиме $(i=1)$ и внешнем повиве провода $(i=2)$ от продольной координаты $x$. Изменение растягивающей силы во внутренней части провода (ядре) вдоль координаты $x$ показано на рис. $10, a$. На рис. 10, $\sigma$ показаны распределения давлений $p^{(i)}$ между зажимом и внешним повивом провода $(i=1)$, а также между внешним повивом провода и его внутренней частью $(i=2)$.

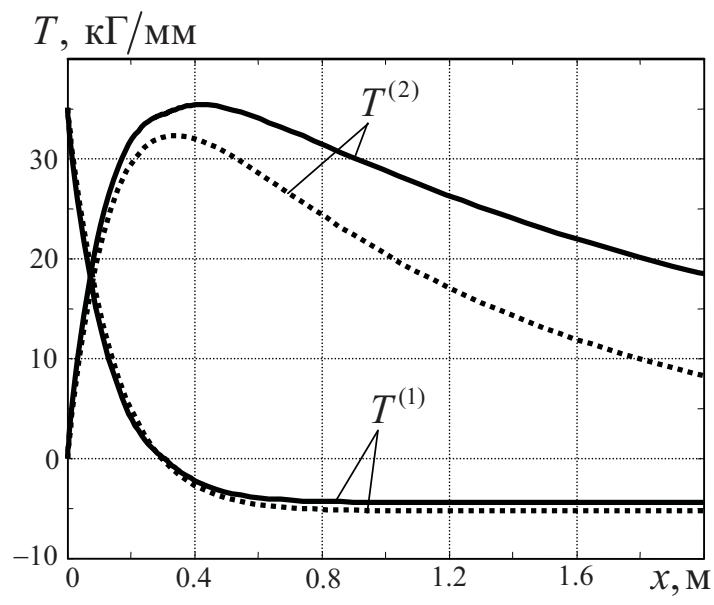

$a$

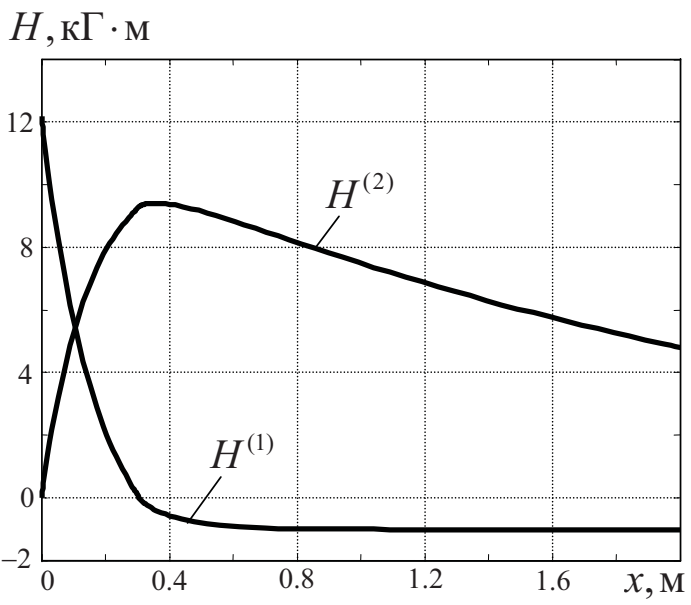

6

Рис. 9. Изменение продольных усилий $T^{(i)}(a)$ и крутящих моментов $H^{(i)}($ б) в зажиме $(i=1)$ и внешнем повиве провода $(i=2)$ вдоль координаты $x$

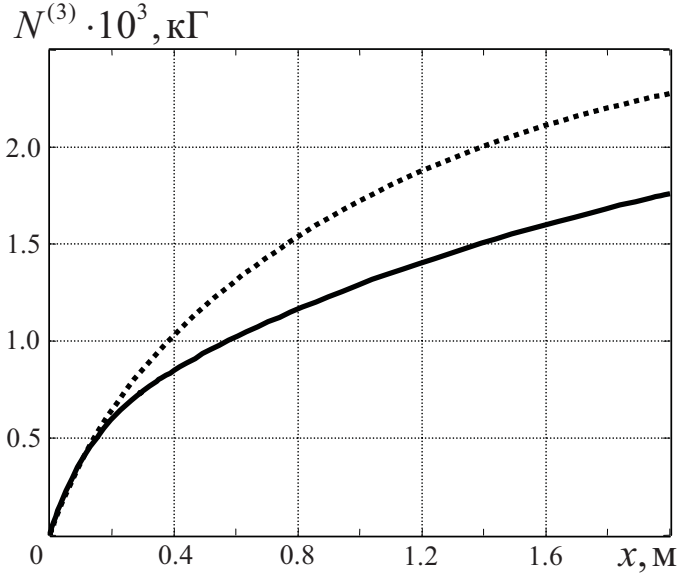

$a$

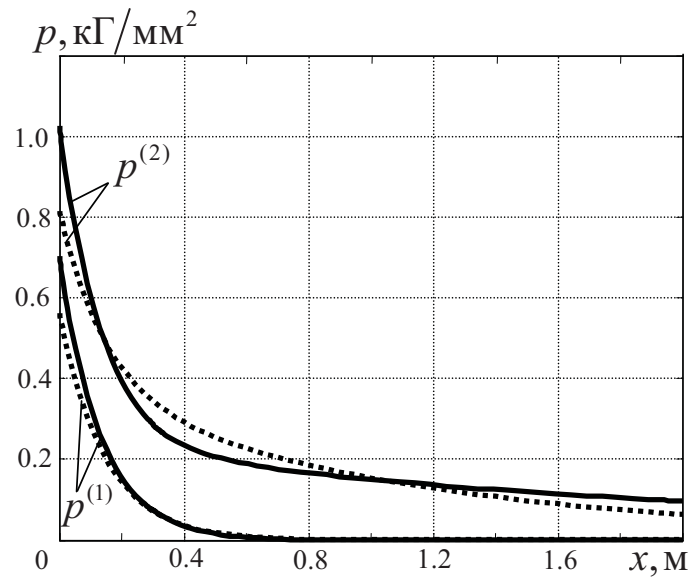

$\sigma$

Рис. 10. Изменение растягивающей силы во внутренней части провода $N^{(3)}(a)$ и внутренних давлений $p^{(i)}(б)$ между зажимом и внешним повивом провода $(i=1)$, а также между внешним повивом провода и его внутренней частью $(i=2)$ вдоль координаты $x$ 

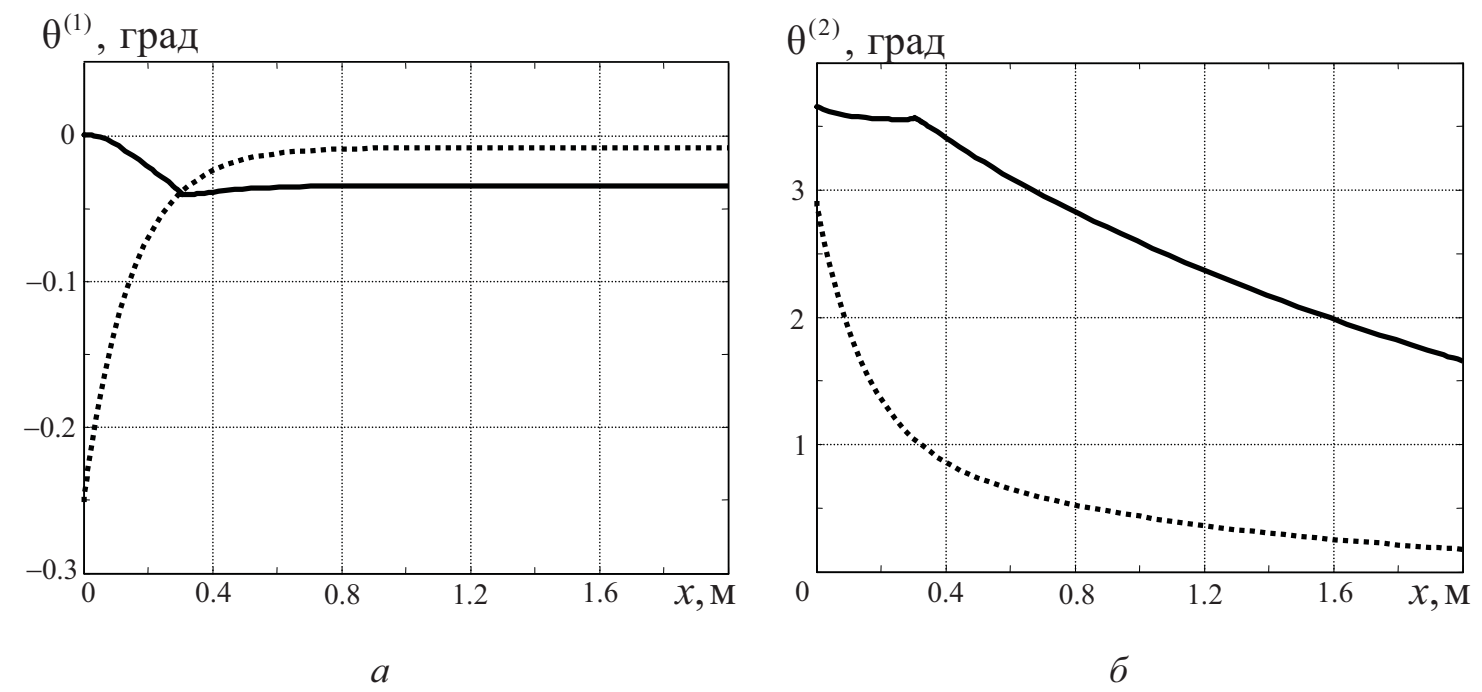

Рис. 11. Изменение углов поворота поперечных сечений зажима $\theta^{(1)}(a)$ и поперечных сечений внешнего повива провода $\theta^{(2)}$ (б) вдоль координаты $x$

На рис. 11 показаны распределения по продольной координате $x$ углов поворота $\theta^{(i)}$ поперечных сечений зажима $(i=1)$ и верхнего повива провода $(i=2)$ относительно оси $x$.

На рис. 9-11 сплошные линии представляют собой решение начальной задачи (45), (48), когда поворот зажима запрещен (случай жесткого крепления). Пунктирные линии соответствуют начальной задаче (45), (46), когда зажим на опоре может поворачиваться свободно.

Начальная величина усилия в зажиме вычислялась как $T_{0}^{(1)}=N_{0}^{(1)} /\left(2 \pi r^{(1)}\right)$, где сила $N_{0}^{(1)}$ принималась равной 2300 кГ.

\section{Выводы}

1. Разработан подход к моделированию многоповивных (многослойных) проволочных конструкций регулярной структуры с учетом взаимодействия проволочных повивов силами давления и трения. Каждый повив представляется с позиции энергетического осреднения как эквивалентная по упругим свойствам анизотропная цилиндрическая оболочка, а сама проволочная конструкция рассматривается как система вложенных друг в друга цилиндрических оболочек, между которыми допускается проскальзывание с учетом сил давления и трения.

2. На основе разработанного подхода получены формулы для вычисления матриц жесткости и податливости, позволяющие дать оценки жесткостям провода на кручение и изгиб с учетом его внутреннего строения и взаимодействия повивов между собой.

3. На примере задачи о взаимодействии провода воздушной линии электропередачи с натяжным спиральным зажимом показан способ анализа несущей способности и конструктивной эффективности спиральных зажимов.

Работа выполнена при финансовой поддержке РФФИ (грант № 13-01-00471-а), а также гранта Президента РФ для государственной поддержки молодых российских ученых - кандидатов наук (код проекта МК-6582.2013.8). 


\section{Библиографический список}

1. Бошнякович А.Д. Механический расчет проводов и тросов линий электропередачи. - Л.: Энергия, 1971. - 295 с.

2. Глазунов А.А. Основы механической части воздушных линий электропередачи. Т.1. Работа и расчет проводов и тросов. - М.-Л.: Госэнергоиздат, 1956. - $192 \mathrm{c.}$

3. Cloutier L., Goudreau S., Cardou A. Fatigue of overhead electrical conductors // In EPRI. Transmission Line Reference Book: Wind Induced Conductor Motion: Second Edition. Palo-Alto, CA: Electric Power Research Institute; 2006 and 2009; 1018554; P. 3-1, to 3-56.

4. Costello G.A. Theory of wire rope. - N.Y.: Springer-Verlag, 1997.

5. Feyrer K. Wire ropes: tension, endurance, reliability. - Berlin, New York: Springer-Verlag, 2007.

6. Pilkey W.D. Analysis and Design of Elastic Beams. Computational Methods. - New York: J. Wiley \& Sons, 2002.

7. Dubois H., Lilien J.L., Dal Maso F. A new theory for frequencies computation of overhead lines with bundle conductors // Rev. AIM - Liege. - 1991. - No. 1. - P. 46-62.

8. Papailiou K.O. On the bending stiffness of transmission line conductors // IEEE Transactions on Power Delivery. - 1997. - Vol. 12. - No. 4. - P. 1576-1588.

9. Cardou A., Jolicoeur C. Mechanical models of helical strands // App. Mech. Rev. - 1997. Vol. 50 (1). - P. 1-14.

10. Foti F., Martinelli L. A model for the cyclic biaxial bending of stranded ropes // Abstract in Conference proceedings of the 20th congress of the AIMETA, Bologna, Italy, 12-15 September 2011, p. 240, available at: http://www.integer.it under its Italian title: Un modello per la flessione biassiale ciclica di funi a trefoli.

11. Strain measurements on ACSR conductors during fatigue tests II - Stress fatigue indicators / S. Goudreau, F. Lévesque, A. Cardou, L. Cloutier // IEEE Trans. on Power Delivery. - 2010. Vol. 25 (4). - P. 2997-3006.

12. Hong K-J., Der Kiuregian A., Sackman J. L. Bending behavior of helically wrapped cables // ASCE J. Eng. Mech. - 2005. - Vol. 131 (5). - P. 500-511.

13. Fekr M.R., McClure G., Farzaneh M. Application of ADINA to stress analysis of an optical ground wire // Computers \& Structures. - 1999. - Vol. 72. - P. 301-316.

14. Finite element model of the contact between a vibrating conductor and a suspension clamp / F. Lévesque, S. Goudreau, L. Cloutier, A. Cardou // Tribology International. - 2011. - Vol. 44 (9). P. 1014-1023.

15. Rawlins C.B. Flexure of a single-layer tensioned cable at a rigid support $/ /$ Proc. $6^{\text {th }}$ International Symposium on Cable Dynamics. - Charleston (U.S.A), 19-22 Sept. 2005. - P. 363-370.

16. Rawlins C.B. Analytical Elements of Overhead Conductor Fabrication // Fultus Corporation. 2005.

17. Rawlins C.B. Flexural self-damping in overhead electrical transmission conductors // J. of Sound and Vib. - 2009. - Vol. 323 (1-2). - P. 232-256.

18. Shalashilin V.I., Danilin A.N., Volkov-Bogorodskiy D.B. Model of overhead line conductor with interaction of layers // Proc. $6^{\text {th }}$ International Symposium on Cable Dynamics. - Charleston (U.S.A). 19-22 Sept. 2005. - P. 371-377.

19. Колебания проводов воздушных линий под воздействием ветра: учеб.-справ. пособие. Ч. 1. Усталостная прочность. Вибрация / под ред. А.А. Виноградова. - М.: Электросетьстройпроект, 2005. - $185 \mathrm{c}$.

20. Новая модель деформирования проволочных систем спиральной структуры / А.Н. Данилин, Е.Л. Кузнецова, Л.Н. Рабинский, С.С. Тарасов // Нелинейный мир. - 2011. - Т. 9, № 10. C. $635-645$.

21. Виноградов А.А., Данилин А.Н., Рабинский Л.Н. Деформирование многослойных проволочных конструкций спирального типа. Математическое моделирование, примеры использования. - М.: Изд-во МАИ, 2014. - 168 с. 
22. Аносов Ю.В., Данилин А.Н., Курдюмов Н.Н. О жесткостях проволочных конструкций спирального типа [Электронный ресурс] // Труды МАИ. - 2015. - № 80. - URL: www.mai.ru/science/trudy/published.php.

23. Виноградов А.А., Рыжов С.В., Штельмах А.А. О разработке методики расчета натяжных спиральных зажимов // Энерг. стр-во. - 1994. - № 3. - С. 60-61.

24. Рыжов С.В. Методика расчета выходных параметров натяжных зажимов спирального типа // Электрические станции. - 1998. - № 1. - С. 8-11.

25. Виноградов А.А., Рыжов С.В., Тищенко А.В. Разработка и освоение промышленного выпуска арматуры спирального типа для подвески проводов // Электрические станции. - 1998. № 1. - C. 3-11.

26. Рыжов С.В., Цветков Ю.Л. Опыт применения арматуры спирального типа на воздушных ЛЭП // ЭЛЕКТРО. - 2005. - № 2. - С. 32-36.

27. Спиральная линейная арматура для подвески и ремонта проводов воздушных ЛЭП. Многочастотные гасители вибрации. Приспособления и устройства для проведения монтажных работ на ВЛ: каталог. Вып. 14 (XI.2004). - М.: Электросетьстройпроект, 2004. - С. 50.

28. Спиральная линейная арматура для подвески и ремонта проводов и грозозащитных тросов ВЛ. Монтажные устройства и приспособления: каталог. Вып. 18. - М.: Электросетьстройпроект, 2011. - С. 72.

29. Спиральная арматура для ВЛ. Технические требования. Стандарт организации ОАО «ФСК ЕЭС». - 2010. - $13 \mathrm{c}$.

30. Preformed Line Products (PLP). Energy Product Catalog, 2007. - 472 p.

\section{References}

1. Boshniakovich A.D. Mekhanicheskii raschet provodov i trosov linii elektroperedachi [Mechanical calculation of wires and cables of power lines]. Leningrad: Energiia, 1971. 295 p.

2. Glazunov A.A. Osnovy mekhanicheskoi chasti vozdushnykh linii elektroperedachi. T. 1. Rabota i raschet provodov i trosov [Fundamentals of mechanical overhead power lines. Vol. 1. The work and the calculation of wires and cables]. Moscow-Leningrad: Gosenergoizdat, 1956. 192 p.

3. Cloutier L., Goudreau S., Cardou A. Fatigue of overhead electrical conductors. In EPRI. Transmission Line Reference Book: Wind Induced Conductor Motion: Second Edition. Palo-Alto, CA: Electric Power Research Institute, 2006 and 2009, 1018554, pp. 3-1, to 3-56.

4. Costello G.A. Theory of wire rope. N.Y.: Springer-Verlag, 1997.

5. Feyrer K. Wire ropes: tension, endurance, reliability. Berlin, New York: Springer-Verlag, 2007.

6. Pilkey W.D. Analysis and Design of Elastic Beams. Computational Methods. New York: J.Wiley \& Sons, 2002.

7. Dubois H., Lilien J.L., Dal Maso F. A new theory for frequencies computation of overhead lines with bundle conductors. Rev. AIM, Liege, 1991, no. 1, pp. 46-62.

8. Papailiou K.O. On the bending stiffness of transmission line conductors. IEEE Transactions on Power Delivery, 1997, vol. 12, no. 4, pp. 1576-1588.

9. Cardou A., Jolicoeur C. Mechanical models of helical strands. App. Mech. Rev., 1997, vol. 50, no. 1, pp. 1-14.

10. Foti F., Martinelli L. A model for the cyclic biaxial bending of stranded ropes. Abstract in Conference proceedings of the 20th congress of the AIMETA. Bologna, Italy, 12-15 Sept. 2011, p. 240, available at: http://ww2.integer.it under its Italian title: Un modello per la flessione biassiale ciclica di funi a trefoli.

11. Goudreau S., Lévesque F., Cardou A., Cloutier L. Strain measurements on ACSR conductors during fatigue tests II - Stress fatigue indicators. IEEE Trans. on Power Delivery, 2010, vol. 25, no. 4, pp. 2997-3006. 
12. Hong K-J., Der Kiuregian A., Sackman J.L. Bending behavior of helically wrapped cables. ASCE J. Eng. Mech., 2005, vol. 131, no. 5, pp. 500-511.

13. Fekr M.R., McClure G., Farzaneh M. Application of ADINA to stress analysis of an optical ground wire. Computers \& Structures, 1999, vol. 72, pp. 301-316.

14. Lévesque F., Goudreau S., Cloutier L., Cardou A. Finite element model of the contact between a vibrating conductor and a suspension clamp. Tribology International, 2011, vol. 44 (9), pp. 1014-1023.

15. Rawlins C.B. Flexure of a single-layer tensioned cable at a rigid support. Proceeding $6^{\text {th }}$ International Symposium on Cable Dynamics, Charleston (U.S.A), 19-22 Sept. 2005, pp. 363-370.

16. Rawlins C.B. Analytical Elements of Overhead Conductor Fabrication. Fultus Corporation, 2005.

17. Rawlins C.B. Flexural self-damping in overhead electrical transmission conductors. J. of Sound and Vib., 2009, vol. 323, no. 1-2, pp. 232-256.

18. Shalashilin V.I., Danilin A.N., Volkov-Bogorodskiy D.B. Model of overhead line conductor with interaction of layers. Proceeding $6^{\text {th }}$ International Symposium on Cable Dynamics. Charleston (U.S.A), 19-22 Sept. 2005, pp. 371-377.

19. Kolebaniia provodov vozdushnykh linii pod vozdeistviem vetra. Part 1, Ustalostnaia prochnost'. Vibratsiia [Training and Reference Manual. Part 1: Fatigue strength. Vibration], ed. by A.A. Vinogradov. Moscow: Elektroset'stroiproekt, 2005. 185 p.

20. Danilin A.N., Kuznetsova E.L., Rabinskii L.N., Tarasov S.S. Novaia model' deformirovaniia provolochnykh sistem spiral'noi struktury [The new model of deformation of the wire system of the spiral structure]. Nelineinyi mir, 2011, vol. 9, no. 10, pp. 635-645.

21. Vinogradov A.A., Danilin A.N., Rabinskii L.N. Deformirovanie mnogosloinykh provolochnykh konstrukcii spiral'nogo tipa. Matematicheskoe modelirovanie, primery ispol'zovaniia [Deformation of multilayer structures spiral wire type. Mathematical modeling, usage examples]. Moscovskii aviatsionnyi institut, 2014. $168 \mathrm{p}$.

22. Anosov Iu.V., Danilin A.N., Kurdiumov N.N. O zhestkostiakh provolochnykh konstruktsii spiral'nogo tipa [The cruelty of the wires spiral type]. Trudy MAI, 2015, no. 80, available at: www.mai.ru/science/trudy/published.php

23. Vinogradov A.A., Ryzhov S.V., Shtel'makh A.A. O razrabotke metodiki rascheta natiazhnykh spiral'nykh zazhimov [On the development of methodology for calculating spiral tension clamps]. Energeticheskoe sroitel'stvo, 1994, no. 3, pp. 60-61.

24. Ryzhov S.V. Metodika rascheta vykhodnykh parametrov natiazhnykh zazhimov spiral'nogo tipa [Methods of calculating the output parameters of the helical-type tension clamps]. Elektricheskie stantsii, 1998, no. 1, pp. 8-11.

25. Vinogradov A.A., Ryzhov S.V., Tishchenko A.V. Razrabotka i osvoenie promyshlennogo vypuska armatury spiral'nogo tipa dlia podveski provodov [Design and development of industrial production of spiral-type valves for the suspension wires]. Elektricheskie stantsii, 1998, no. 1, pp. 3-11.

26. Ryzhov S.V., Tsvetkov Iu.L. Opyt primeneniia armatury spiral'nogo tipa na vozdushnykh LEP [Experience with reinforcement spiral-type overhead lines]. ELEKTRO, 2005, no. 2, pp. 32-36.

27. Spiral'naia lineinaia armatura dlia podveski i remonta provodov vozdushnykh LEP. Mnogochastotnye gasiteli vibratsii. Prisposobleniia i ustroistva dlia provedeniia montazhnykh rabot na VL. Katalog, iss. 14 (XI.2004) [Multi-frequency vibration dampers. Tools and devices for carrying out installation work on the overhead line. Catalog. Iss. 14 (XI.2004)]. Moscow: Elektroset'stroiproekt, 2004. $50 \mathrm{p}$.

28. Spiral'naia lineinaia armatura dlia podveski i remonta provodov i grozozashchitnykh trosov VL. Montazhnye ustroistva i prisposobleniia. Katalog, iss. 18 [The spiral line fittings for suspension and repair wire and ground wire overhead lines. Mounting devices and accessories. Catalog. Issue 18]. Moscow: Elektroset'stroiproekt, 2011. 72 p.

29. Spiral'naia armatura dlia VL. Tekhnicheskie trebovaniia. Standart organizatsii OAO «FSK EES» [Spiral reinforcement for the overhead line. Technical requirements. Standard organization of JSC "FGC UES"]. 2010. 13 p.

30. Preformed Line Products (PLP). Energy Product Catalog, 2007. 472 p. 